\title{
Foreword to the Special Issue on Micro- and Nano-Metrology in Japan (I)
}

\author{
Yuki Shimizu $^{1} \cdot$ Yasuhiro Mizutani $^{2} \cdot$ Masaki Michihata $^{3}$
}

Accepted: 18 February 2021 / Published online: 27 February 2021

(c) International Society for Nanomanufacturing and Tianjin University and Springer Nature Singapore Pte Ltd. 2021

Micro- and nano-metrology occupies an important position in modern production engineering where higher-precision machining is required. A visualization of the process is indispensable for the creation of new technologies in stateof-the-art manufacturing that deals with the geometrical quantity in micrometers/nanometers. Precision micro- and nano-metrology is thus becoming more and more important as the platform of various manufacturing technologies, as well as strategic tools for responding to rapidly changing fabrication techniques in many scientific and industrial fields.

In Japan, the subcommittee entitled "Intelligent NanoMeasure" was established in 2004 by pioneers in the Japan Society for Precision Engineering (JSPE). Following this subcommittee, the Technical Committee (TC) of Intelligent Nano-Measure was established in 2006. This TC explores a wide range of measurement technologies in manufacturing including basic research that pursues the continuous evolution of measurement standards, as well as the applied research for the development of new measurement technologies applicable to new manufacturing technologies. Major academic researchers and engineers related to micro- and nano-metrology and manufacturing in Japan are participating in the TC, and are actively engaged in activities. This special issue was compiled by three young researchers

Yuki Shimizu

yuki.shimizu.d2@tohoku.ac.jp

Yasuhiro Mizutani

mizutani@mech.eng.osaka-u.ac.jp

Masaki Michihata

michihata@nanolab.t.u-tokyo.ac.jp

1 Department of Finemechanics, Tohoku University, Sendai 9808579, Japan

2 Department of Mechanical Engineering, Osaka University, Suita 5650871, Japan

3 Department of Precision Engineering, The University of Tokyo, Bunkyo 1138654, Japan belonging to this TC; Dr. Yuki Shimizu of Tohoku University, Dr. Yasuhiro Mizutani of Osaka University, and Dr. Masaki Michihata of the University of Tokyo as guest editors. In addition, this special issue also contains a large number of papers submitted by young researchers who belong to the TC of Intelligent Nano-Measure.

A series of papers in the special issue is published in Nanomanufacturing and Metrology in two issues. The first issue includes four original research papers treating inprocess diameter measurement of a micro-optical fiber on the basis of standing wave illumination, ghost imaging for position mapping of a weakly scattered light source with the enhancement of the technique of deep learning, the evaluation of internal residual stress in plastic parts based on terahertz spectroscopy, and design and development of a threeaxis miniature stage with closed-loop control and reduced motion errors. One review article treating laser interference lithography for the fabrication of two-dimensional grating pattern structures for optical micro- and nano-metrology is also included in the first issue.

The guest editors would like to take this opportunity to thank all the contributing authors for their great efforts in presenting their excellent research work for the special issue. Our appreciation also goes to all the experts in the Technical Committee of Intelligent Nano-Measure in JSPE who have provided invaluable comments and suggestions for the further improvement of the quality of the research papers. The guest editors would also like to thank Professor Wei Gao (Tohoku University, Japan) for the tremendous support in the preparation of this special issue. Kind support from the editorial staff of Nanomanufacturing and Metrology is also appreciated. 


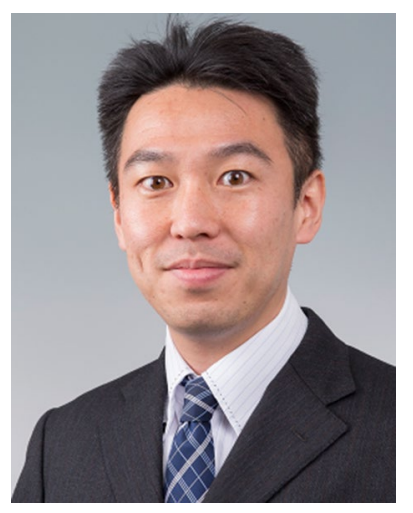

Yuki Shimizu received his MS in precision engineering from Tohoku University, Japan, in 2002. He had spent his career at Hitachi Ltd. from 2002 to 2011 and had been involved in the research on head-disk interface technology for a hard disk drive. $\mathrm{He}$ received his $\mathrm{PhD}$ in Mechanical Engineering from Nagoya University, Japan, in 2009. He has been an associate professor in the Department of Finemechanics, Tohoku University, Japan, since 2011. He has published more than 150 journal papers, including four review papers. He was awarded the title of Distinguished Researcher at Tohoku University in 2020. His research interest includes precision dimensional metrology and optical metrology. $\mathrm{He}$ is a member of the Japan Society for Precision Engineering (JSPE) and the Japan Society for Mechanical Engineers (JSME).

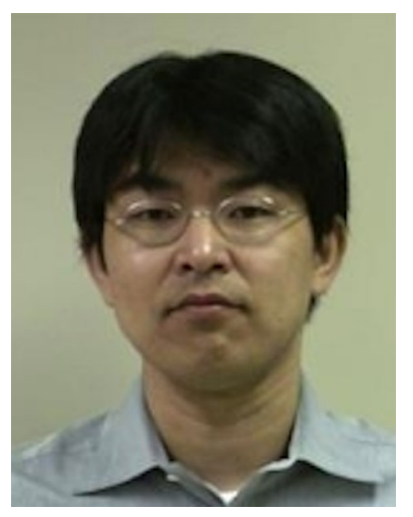

Yasuhiro Mizutani, PhD obtained his PhD degree in Mechanical Engineering from Tokyo University of Agriculture \& Technology, Japan, in 2008. He has also $\mathrm{BE}$ and ME degrees in Nuclear Engineering from Osaka University in 1997 and 1999, respectively. From 1999 to 2003, he joined a researcher at Panasonic Corporation. Then, from 2003 to 2009 , he worked as a research associate in the Department of Mechanical Systems Engineering at Tokyo University of
Agriculture \& Technology, Japan. He joined the University of Tokushima, Japan, in 2009 as an associate professor. He is currently an associate professor at Osaka University since 2015. Professor Mizutani's research interests include interferometry, polarimetry, 3D surface measurement, optical trapping, and 3D lithography. In these areas, he has published over 100 papers in refereed international journals and conferences. He is a member of SPIE and OSA.

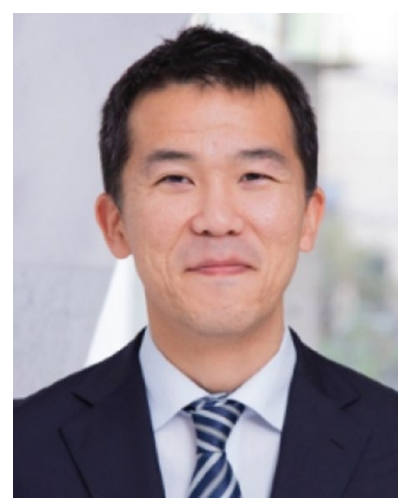

Masaki Michihata is currently an associate professor at the Department of Precision Engineering, the University of Tokyo. He received his $\mathrm{BS}, \mathrm{MS}$, and $\mathrm{PhD}$ degrees in Mechanical Engineering from Osaka University in 2004, 2007, and 2010, respectively. He has authored more than 50 journal papers in optical dimensional measurement and processing. His current research interests include three-dimensional metrology, optical resonance-based dimensional measurement, in-process/on-machine measurements, and optical-radiation-pressure-based nano-fabrication. $\mathrm{He}$ is a member of the JSPE and JSME. 